\title{
Anatomical and reverse shoulder arthroplasty utilizing a single implant system with a platform stem: \\ A prospective observational study with midterm follow-up
}

\author{
Lindsay Flynn', Matthew R Patrick', Christopher Roche², \\ Joseph D Zuckerman ${ }^{3}$, Pierre-Henri Flurin ${ }^{4}$, Lynn Crosby ${ }^{5}$, \\ Richard Friedman ${ }^{6}$ and Thomas W Wright ${ }^{1}$
}

\begin{abstract}
Background: No studies compare outcomes of anatomic total shoulder arthroplasty to reverse total shoulder arthroplasty with more than five-year follow-up.

Methods: A multicenter prospectively collected shoulder registry was utilized to review all patients undergoing primary anatomic total shoulder arthroplasty or primary reverse total shoulder arthroplasty with a minimum five-year follow-up utilizing a single platform stem implant system. One-hundred-ninety-one patients received an anatomic total shoulder arthroplasty and 139 patients received a reverse total shoulder arthroplasty. Patients were scored preoperatively and at latest follow-up using the simple shoulder test (SST), University of California Los Angeles (UCLA), American shoulder and elbow surgeons (ASES), Constant, and shoulder pain and disability index (SADI) scores as well as range of motion. Radiographs were evaluated for implant loosening or notching. Complications were reviewed. A Student's two-tailed, unpaired t-test identified differences in preoperative, postoperative, and pre-to-postoperative improvements.

Results: Reverse total shoulder arthroplasty patients were significantly older than anatomic total shoulder arthroplasty patients. All patients demonstrated significant improvement in functional metric scores and range of motion following anatomic total shoulder arthroplasty or reverse total shoulder arthroplasty. There was no difference in final outcome scores between anatomic total shoulder arthroplasty and reverse total shoulder arthroplasty patients at midterm followup; however, reverse total shoulder arthroplasty patients demonstrated significantly less motion.

Discussion: We demonstrate equivalent outcomes with five scoring metrics at mean follow-up of $7 \mathrm{I} .3 \pm \mathrm{I4}$.I months. Although postoperative scores were significantly greater than preoperative scores for both anatomic total shoulder arthroplasty and reverse total shoulder arthroplasty patients, significant differences in outcome scores between cohorts were not observed.
\end{abstract}

\section{Keywords}

total shoulder arthroplasty, reverse total shoulder arthroplasty, rotator cuff arthropathy, shoulder osteoarthritis, shoulder range of motion, radiographic evaluation

Date received: 24th July 2018; revised: 5th March 2019; accepted: 6th March 2019

\footnotetext{
'Department of Orthopaedics and Rehabilitation, University of Florida, Gainesville, USA

${ }^{2}$ Exactech, Inc., Gainesville, USA

${ }^{3}$ NYU Center for Musculoskeletal Care, NYU Langone Medical Center, New York, USA

${ }^{4}$ Bordeaux Merignac Sport Clinic, Mérignac, France
}

\footnotetext{
${ }^{5}$ Department of Orthopaedics, Medical College of Georgia, Augusta, USA

${ }^{6}$ Medical University of South Carolina, Charleston, USA

Corresponding author:

Thomas W Wright, Orthopaedics and Sports Medicine Institute, University of Florida, 3450 Hull Road, Gainesville, FL 326II, USA. Email:wrightw@ortho.ufl.edu
} 


\section{Introduction}

Shoulder arthroplasty has become a reliable and widely used treatment for glenohumeral arthropathy. Reverse total shoulder arthroplasty (rTSA) is most commonly indicated for the treatment of glenohumeral arthritis in patients with severe rotator cuff insufficiency, although the indications are expanding to include complex fractures, revisions, and primary arthritis in elderly patients. The usage of both anatomic total shoulder arthroplasty (aTSA) and rTSA has increased significantly worldwide due, in part, to the predictability of acceptable outcomes achieved with each prosthesis type for its respective indications, but also due to the aging population and the increased use of the rTSA. ${ }^{1-6}$ As surgeons are expanding the indications for rTSA, there is an increasing percentage of primary arthroplasty with rTSA. ${ }^{2}$ Although patients requiring aTSA are typically less impaired than patients requiring rTSA, both groups have previously shown significant improvement after shoulder arthroplasty in short-term follow-up. ${ }^{7}$

Whereas several studies have published data on survivorship of both aTSA and rTSA, few have compared outcomes between the two treatments; and those that do only utilize short-term data. ${ }^{1,7,8}$ The purpose of this study is to compare patients undergoing either primary rTSA or aTSA with a minimum of five-year follow-up with multiple functional metric scores, quantitative range of motion (ROM), and radiographic evaluation.

The authors hypothesize that, similar to short-term outcome studies, both cohorts will see significant improvements in pain and function following treatment with either aTSA or rTSA. With this midterm data, the authors hypothesize that there will be a statistical difference in the magnitude of change of pre- to postoperative functional metric scores and all ROM measurements in the rTSA cohort compared to the aTSA cohort.

\section{Materials and methods}

A multicenter prospectively collected shoulder arthroplasty registry was utilized. Preoperative and postoperative data were analyzed from 330 patients (average age: $69.3 \pm 8.3 \mathrm{yrs} ; \min =36 \quad \mathrm{yrs}$ and $\max =89 \mathrm{yrs}$ ) who received a primary aTSA or primary rTSA with a minimum of five-year follow-up (average follow-up $=71.3 \pm 14.4$ months) utilizing the same implant system that incorporates a platform stem (Exactech Equinoxe, Gainesville, Florida). One-hundred-ninety-one patients with an average age of $67.0 \pm 8.8$ years $(\mathrm{min}=36 \mathrm{yrs}$ and $\max =86 \mathrm{yrs}$ ), 103 females (average: $69.0 \mathrm{yrs}$ ), and 88 males (average: $64.6 \mathrm{yrs}$ ) received a primary aTSA shoulder for treatment of osteoarthritis (OA), post-traumatic arthritis, or inflammatory arthropathy. One-hundred-thirtynine patients with an average age of $72.5 \pm 6.4$ years $(\min =55 \mathrm{yrs}$ and $\max =89 \mathrm{yrs}), 96$ females (average: $73.3 \mathrm{yrs}$ ), and 43 males (average 70.8 yrs) received a primary rTSA. In 83 cases the indication was rotator cuff arthropathy, rotator cuff tear in 14, primary OA in 17 , inflammatory arthropathy in 8 , osteonecrosis in 7 , acute fractures in 4 , malunion in 2 , and post-traumatic $\mathrm{OA}$ in 4.

Nonaugmented, all-polyethylene glenoid components were used for aTSA procedures. Sixty-five arthroplasties utilized a keeled glenoid component and 126 arthroplasties utilized a pegged glenoid component. All aTSA glenoid components were secured using polymethyl methacrylate bone cement. For rTSA cases, a standard, nonaugmented glenoid baseplate was utilized for all procedures.

The patients were evaluated and scored preoperatively and at latest follow-up using the simple shoulder test (SST), University of California Los Angeles (UCLA), American shoulder and elbow surgeons (ASES), Constant, and shoulder pain and disability index (SADI) scoring metrics. Additionally, the patients' active abduction, active forward flexion, active external rotation, and passive external rotation were also measured preoperatively and at latest follow-up. Internal rotation was measured by vertebral segments and was scored by the following discrete assignment: $0^{\circ}=0$, hip $=1$, buttocks $=2$, sacrum $=3, \quad$ L5-L4 $=4, \quad$ L3$\mathrm{L} 1=5$, $\mathrm{Th} 12-\mathrm{Th} 8=6$, and $\mathrm{Th} 7$ or higher $=7$.

The average follow-up for aTSA patients was $73.8 \pm 16.3$ months and the average follow-up for rTSA patients was $67.8 \pm 10.5$ months. Improvements in outcome using each metric score were normalized on a 100-point scale, correlated, and compared. Radiographs were evaluated for evidence of implant loosening or notching. Complications were reviewed and recorded. A Student's two-tailed, unpaired t-test was used to identify differences in preoperative, postoperative, and preoperative-to-postoperative improvements, where $\mathrm{p}<0.05$ denoted a significant difference.

\section{Results}

All patients demonstrated significant improvements in pain and function following treatment with aTSA (Table 1) and rTSA (Table 2). rTSA patients were on average significantly older (72.5 versus 67.0 yrs; $\mathrm{p}<0.0001)$ and weighed significantly less (165.7 versus $182.3 \mathrm{lbs} ; \mathrm{p}<0.0001)$ than aTSA patients. aTSA patients had significantly longer average followup than rTSA patients (73.8 versus 67.8 months; $\mathrm{p}=0.0019)$. Ten complications $(5.2 \%)$ occurred in the aTSA cohort, and eight complications $(5.8 \%)$ occurred in the rTSA cohort. The complication rate between 

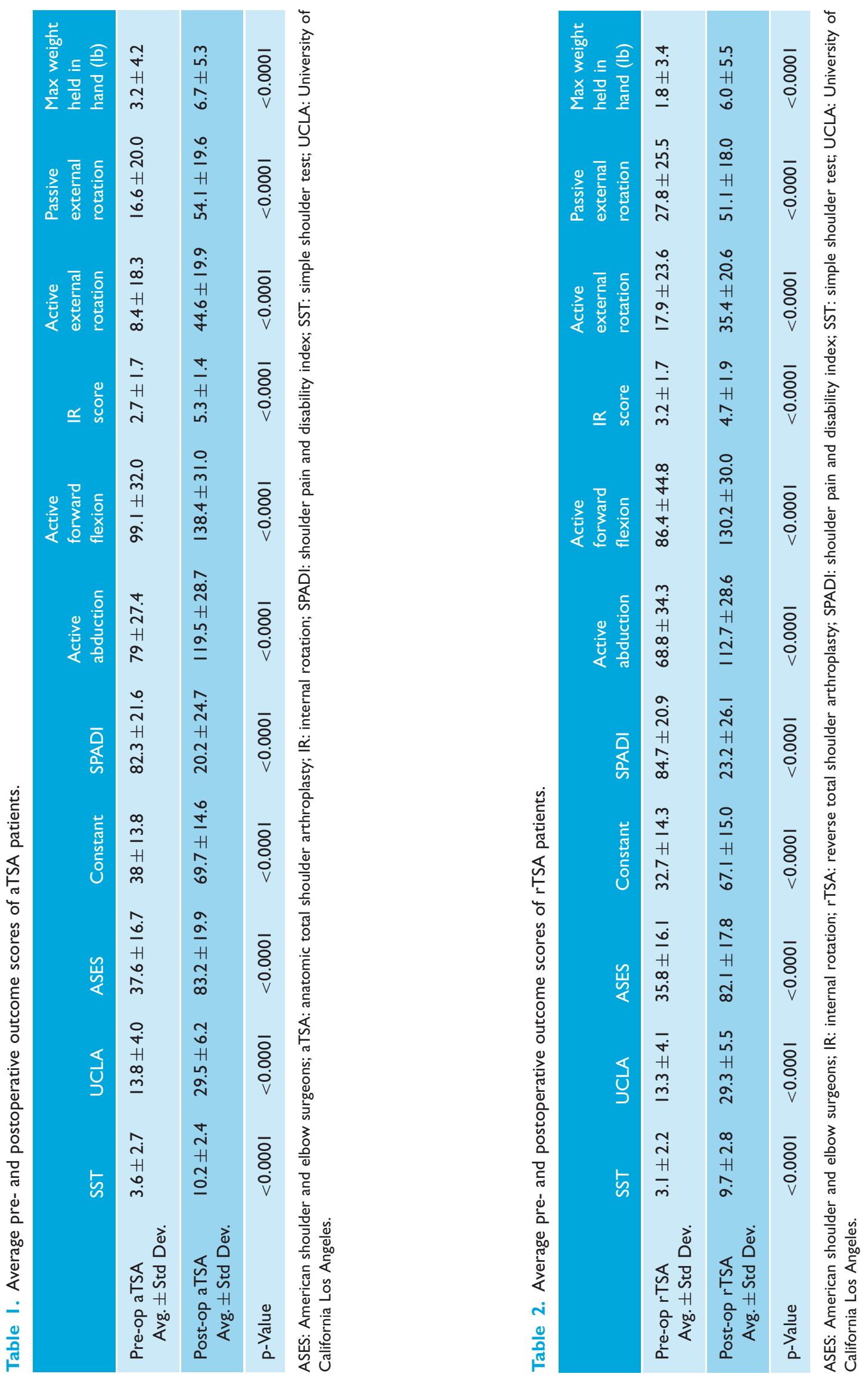


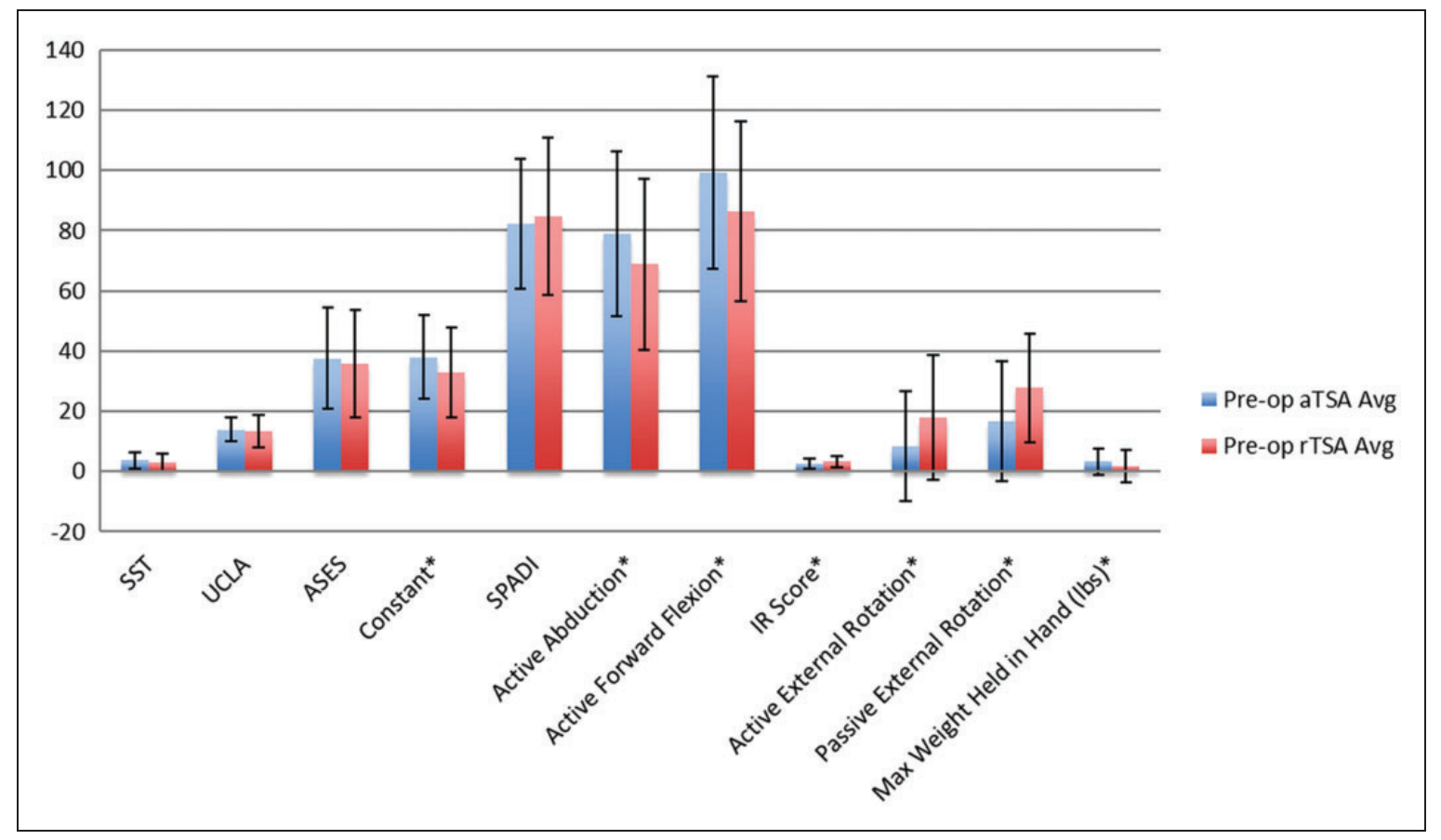

Figure I. Comparison of average preoperative outcome scores of aTSA versus rTSA patients. ASES: American shoulder and elbow surgeons; aTSA: anatomic total shoulder arthroplasty; IR: internal rotation; rTSA: reverse total shoulder arthroplasty; SPADI: shoulder pain and disability index; SST: simple shoulder test; UCLA: University of California Los Angeles.

aTSA and rTSA patients was not statistically different. Radiographic outcomes were available for 161 of 191 aTSA patients $(84.3 \%) ; 38.5 \%$ of these patients had a radiolucent line on their glenoid component with an average radiolucent line grade of 0.88 (99 grade 0,25 grade 1,11 grade 2 , and 15 grade 3, 6 grade 4 , and 5 grade 5) based on the Lazarus grading system. ${ }^{9}$ Humeral radiolucencies were present in $4.3 \%$ of aTSA patients. Radiographic outcomes were available for 119 of 139 rTSA patients $(85.6 \%)$; these patients had a scapular notching rate of $19.3 \%$ with an average scapular notching grade of 0.28 (96 grade 0,15 grade 1,12 grade 2, and 2 grade 3 ) based on the Sirveaux classification system. ${ }^{10}$ Humeral radiolucencies were present in $5.0 \%$ of rTSA patients. The humeral radiolucency rate between aTSA and rTSA patients was not statistically different.

There were 18 complications reported. Of the 10 complications reported in the aTSA cohort, five required surgical intervention. The most commonly reported complication was RTC, occurring in three patients. Of the eight complications reported in the rTSA cohort, only two required surgical intervention. The most commonly reported complication was acromion/scapular spine fractures, occurring in three patients, all of whom were treated nonoperatively. All periprosthetic fractures were the result of falls.

Figure 1 compares the preoperative outcomes between the aTSA and rTSA cohorts. As described in
Figure 1, rTSA patients had a significantly worse preoperative Constant score and had significantly less preoperative active abduction, forward flexion, and strength, though they had significantly better active and passive external rotation, as compared to aTSA patients. Figure 2 compares the postoperative outcomes between the aTSA and rTSA cohort. As described in Figure 2, no difference in clinical metric scores was observed between aTSA and rTSA patients at midterm follow-up; however, rTSA patients had significantly less motion as measured by four of the six ROM (active abduction, forward flexion, and active and passive external rotation) measurements. Figure 3 compares the preoperative-to-postoperative improvement in outcomes between the aTSA and rTSA cohort. As described in Figure 3, no difference in improvement was observed with any clinical metric scores between aTSA and rTSA patients at midterm follow-up; however, rTSA patients had significantly less improvement in motion as measured by three of the six ROM (internal rotation score, active, and passive external rotation) measurements.

\section{Discussion}

The results of this study demonstrate significant improvements in pain and function following treatment with both aTSA and rTSA utilizing a single platform shoulder system at a mean follow-up of $71.3 \pm 14.1$ 


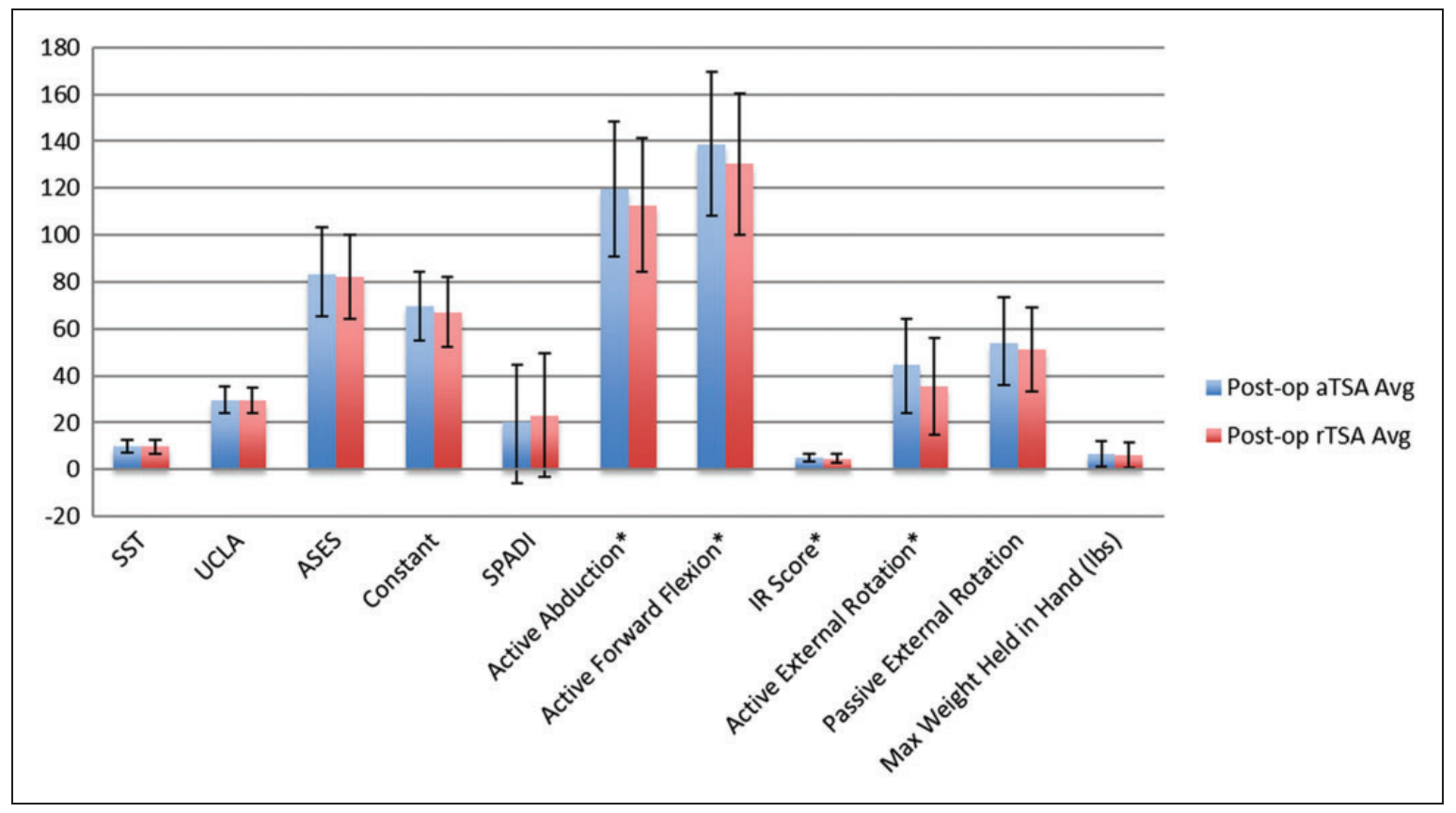

Figure 2. Comparison of average postoperative outcome scores of aTSA versus rTSA patients. ASES: American shoulder and elbow surgeons; aTSA: anatomic total shoulder arthroplasty; IR: internal rotation; rTSA: reverse total shoulder arthroplasty; SPADI: shoulder pain and disability index; SST: simple shoulder test; UCLA: University of California Los Angeles.

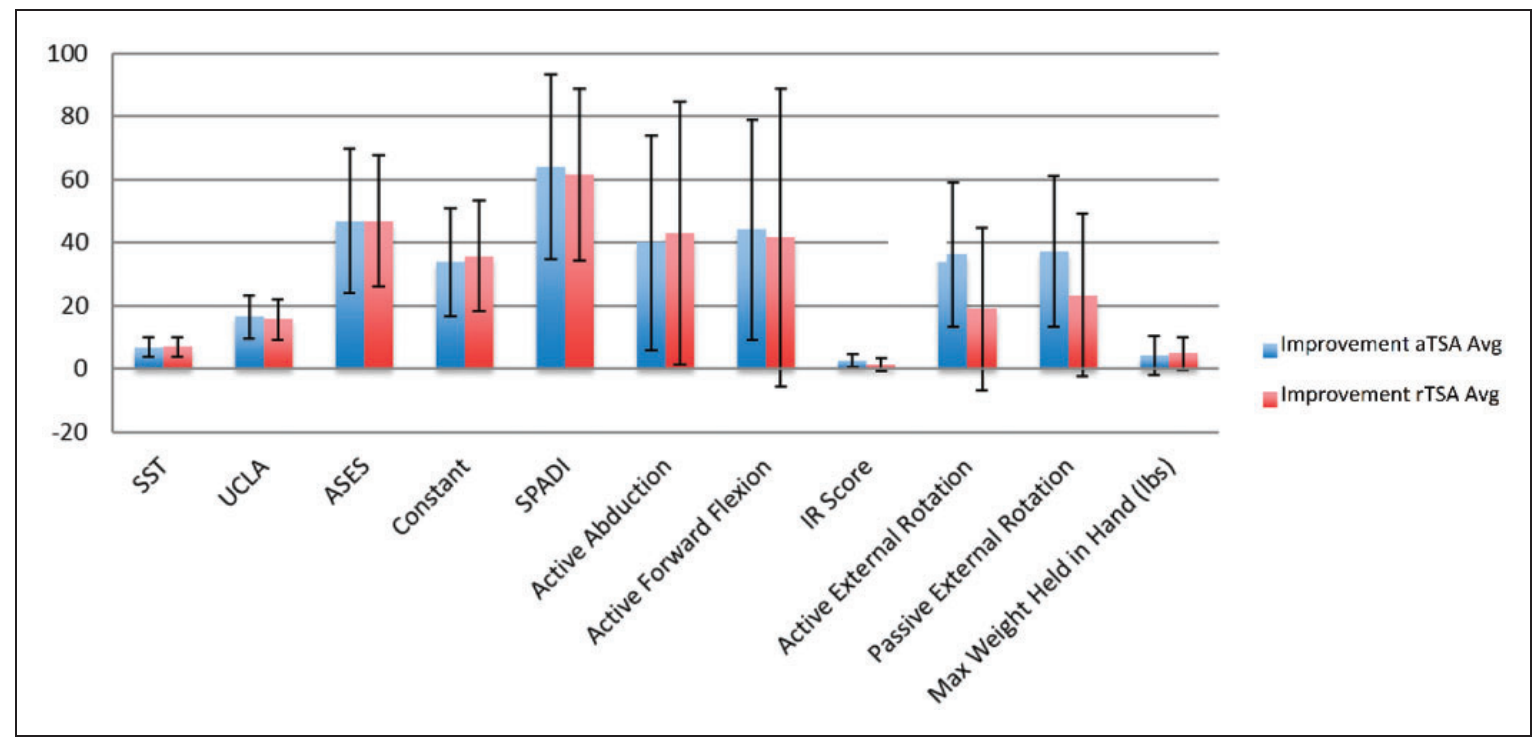

Figure 3. Comparison of average improvement ( $\Delta$ preoperative-to-postoperative outcomes scores) of aTSA versus $r$ TSA patients. ASES: American shoulder and elbow surgeons; aTSA: anatomic total shoulder arthroplasty; IR: internal rotation; rTSA: reverse total shoulder arthroplasty; SPADI: shoulder pain and disability index; SST: simple shoulder test; UCLA: University of California Los Angeles.

months. While aTSA and rTSA were used to treat different preoperative conditions, each treatment method provided significant improvements in all outcome score measurements. Each cohort saw significant improvements in all motion measurements, strength, and pain postoperatively as well.
There were several significant differences between the preoperative data of the two shoulder arthroplasty cohorts. On preoperative evaluations, rTSA patients were older, weighed less, and had less active abduction and active forward flexion, though they had significantly better active and passive external rotation 
compared to aTSA patients. Strength was also greater in the aTSA patients preoperatively. Preoperatively, rTSA patients had a significantly worse Constant score. These preoperative findings are similar to other comparison studies. ${ }^{1,6,11,12-18}$

In both aTSA and rTSA cohorts, the patients had significant improvements in all functional metric scores and ROM pre- to postoperatively. Interestingly, this study did not find a statistical difference in the rTSA versus aTSA magnitude of change of preoperative-topostoperative functional metric scores or ROM, which has been described in previous studies. ${ }^{111}$ This finding may be due to the rTSA cohort's heterogeneity of the preoperative diagnosis. In this study, rTSA patients had preoperative diagnoses of rotator cuff arthropathy, RTC, and OA. There was also no significant difference in improvement in strength in terms of the magnitude of preoperative-to-postoperative improvement in strength between the two cohorts.

One of the more interesting findings is the lack of statistical difference in any of the five postoperative functional metric scores between the aTSA and rTSA groups at minimum five-year follow-up. This finding shows that both the functional measurements and the patients' perception of function are similar between aTSA and rTSA at a minimum of five-year follow-up. While aTSA patients had significantly greater postoperative active abduction, forward flexion, internal rotation, and external rotation, there was no significant difference in passive external rotation between the two groups. Although these slight increases in mean ROM were statistically significant, their clinical significance is unknown. It is doubtful that a difference in forward flexion of $8^{\circ}$, or internal rotation of one anatomic segment, would be noticed by a patient and is likely not clinically meaningful. However, it is possible that an improvement of $9^{\circ}$ in active external rotation favoring an aTSA might be perceived as clinically meaningful by the patient.

Analysis of surgical complications demonstrated no statistically significant difference between the aTSA and rTSA cohorts $(5.2 \%$ versus $5.8 \%)$. The rTSA complication rate in this study is lower than historic reports. ${ }^{1,11,19}$ Earlier reports on rTSA frequently included revision surgeries, minor complications, and notching in the complication results. ${ }^{20}$ The relatively low complication rate for rTSA reported in this large database analysis may be partially explained by the fact that all cases were primary operations, minor complications were not reported, and notching was not included as a complication unless the notching resulted in a surgical intervention (Table 3). The low complication rate can also be attributed to the fact that high volume fellowship trained shoulder arthroplasty surgeons performed the surgeries. Finally, the low complication rate is possibly related to the use of a medial glenoid/lateral humerus prosthesis design that is optimized to minimize scapular notching and maximize ROM and stability. ${ }^{11,21}$

Scapular notching was present in $19.3 \%$ of rTSA with a mean scapular notching grade of 0.28 , which is lower than previous studies that have reported rates of $30-35 \% .{ }^{11,22,23}$ Of the 29 rTSAs with radiographic evidence of notching, 27 were either grade 1 or $2(93 \%)$. There were only two rTSA with grade 3 notching. No grade 4 notches were noted. No patients required a revision due to scapular notching.

There are several limitations of this study. While the shoulder arthroplasty registry utilized is prospectively

Table 3. Complications of aTSA versus rTSA.

\begin{tabular}{|c|c|c|}
\hline & aTSA & rTSA \\
\hline Rotator cuff tear & 3 (I required revision to $r T S A)$ & 0 \\
\hline Symptomatic loose glenoid & 3 (I required revision glenoid) & 0 \\
\hline Instability & I (Required reoperation) & 2 (I required revision) \\
\hline Periprosthetic humerus fracture & I & 2 (I required ORIF) \\
\hline Symptomatic loose humeral stem & I (Required revision stem) & 0 \\
\hline Periprosthetic infection & I (Required reoperation) & 0 \\
\hline Acromion/scapular spine fracture & 0 & 3 \\
\hline Other & 0 & I \\
\hline Total & 10 & 8 \\
\hline
\end{tabular}

aTSA: anatomic total shoulder arthroplasty; rTSA: reverse total shoulder arthroplasty. 
collected, the data were retrospectively reviewed for this study. As a result, there are inherent limitations as with all retrospective studies. It reports on the midterm clinical results of a single platform shoulder system for 330 patients with minimum five-year follow-up. The comparative results may continue to change with time, as the long-term outcome beyond 15 years of the reverse shoulder prosthesis in particular is unknown. ${ }^{24}$ The durability question is a draw between the two implants at a minimum of five-year follow-up. Additionally, registry analyses such as this can contain numerous variables that can limit impact such as multiple centers with different patient populations, different surgeons, different surgery centers, different rehabilitation methods, and different data collection methods. However, the authors have standardized the practices of each center and facilitated the use of standardized data collection forms to quantify outcomes using multiple different scoring metrics. The shoulder registry data are also regularly audited to confirm the quality and completeness of the inputs. Another limitation is that the radiographic analyses are performed at each individual institution by the operating surgeon. Future work could incorporate the use of a single or multiple independent reviews of all radiographs to further minimize bias.

This comparative clinical study of aTSA and rTSA using a single platform shoulder system demonstrates equivalent outcomes with five different scoring metrics at a mean follow-up of $71.3 \pm 14.1$ months. While both aTSA and rTSA cohorts observed significant improvements in all preoperative-to-postoperative functional outcome scores and ROM values, there were no observed statistically significant differences in outcome scores between the two cohorts at final follow-up. aTSA had slightly improved ROM compared to rTSA in this study, but the clinical significance of this is unknown. The complication rates are similar between the two groups, and much lower for rTSA than previously reported. Longer-term follow-up is needed to determine if these results hold up over time.

\section{Declaration of Conflicting Interests}

The author(s) declared the following potential conflicts of interest with respect to the research, authorship, and/or publication of this article: Mr Roche is an employee of Exactech, Inc. Thomas W Wright receives royalties and has a consultancy agreement with Exactech, Inc. Zuckerman is a paid consultant for Exactech, Inc., and receives royalties from Exactech, Inc. Flurin has a consultancy agreement with Exactech, Inc., and receives royalties from Exactech, Inc. Crosby has a consultancy agreement with Exactech, Inc., and receives royalties from Exactech, Inc. Friedman has a consultancy agreement with Exactech, Inc. The University of Florida Department of Orthopaedics and Rehabilitation receives research support from Exactech, Inc. The remaining authors, their immediate families, and any research foundations with which they are affiliated have not received any financial payments or other benefits from any commercial entity related to the subject of this article. IRB Information: WIRB study number 1112376 .

\section{Funding}

The author(s) received no financial support for the research, authorship, and/or publication of this article.

\section{Ethical Review and Patient Consent}

The study was approved by the Western Institutional Review Board, study number 1112376.

\section{Research Material Access}

Please contact Thomas W Wright, Orthopaedics and Sports Medicine Institute, University of Florida, 3450 Hull Road, Gainesville, FL 32611, USA; email, wrightw@ortho.ufl.edu, to request access to underlying research materials. The paper is not based on a previous communication to a society or meeting.

\section{References}

1. Flurin PH, Marczuk Y, Janout M, et al. Comparison of outcomes using anatomic and reverse total shoulder arthroplasty. Bull Hosp Jt Dis 2013; 71: 101-107.

2. Jain NB and Yamaguchi K. The contribution of reverse shoulder arthroplasty to utilization of primary shoulder arthroplasty. J Shoulder Elbow Surg 2014; 23: 1905-1912.

3. Kim SH, Wise BL, Zhang Y, et al. Increasing incidence of shoulder arthroplasty in the United States. J Bone Joint Surg Am 2011; 93: 2249-2254.

4. Mizuno N, Denard PJ, Raiss P, et al. Reverse total shoulder arthroplasty for primary glenohumeral osteoarthritis in patients with a biconcave glenoid. J Bone Joint Surg Am 2013; 95: 1297-1304.

5. Samuelsen BT, Wagner ER, Houdek MT, et al. Primary reverse shoulder arthroplasty in patients aged 65 years or younger. J Shoulder Elbow Surg 2017; 26: e13-e17.

6. Smith CD, Guyver P and Bunker TD. Indications for reverse shoulder replacement: a systematic review. $J$ Bone Joint Surg Br 2012; 94: 577-583.

7. Puskas B, Harreld K, Clark R, et al. Isometric strength, range of motion, and impairment before and after total and reverse shoulder arthroplasty. J Shoulder Elbow Surg 2013; 22: 869-876.

8. Kiet TK, Feeley BT, Naimark M, et al. Outcomes after shoulder replacement: comparison between reverse and anatomic total shoulder arthroplasty. J Shoulder Elbow Surg 2015; 24: 179-185.

9. Lazarus MD, Jensen KL, Southworth C, et al. The radiographic evaluation of keeled and pegged glenoid component insertion. J Bone Joint Surg Am 2002; 84-A: 1174-1182.

10. Lévigne C, Boileau P, Favard L, et al. Scapular notching in reverse shoulder arthroplasty. J Shoulder Elbow Surg 2008; 17: 925-935. 
11. Flurin PH, Roche CP, Wright TW, et al. A comparison and correlation of clinical outcome metrics in anatomic and reverse total shoulder arthroplasty. Bull Hosp Jt Dis 2015; 73: S118-S123.

12. Levy JC, Everding NG, Gil CC, et al. Speed of recovery after shoulder arthroplasty: a comparison of reverse and anatomic total shoulder arthroplasty. J Shoulder Elbow Surg 2014; 23: 1872-1881.

13. Edwards TB, Kadakia NR, Boulahia A, et al. A comparison of hemiarthroplasty and total shoulder arthroplasty in the treatment of primary glenohumeral osteoarthritis. Results of a multicenter study. J Shoulder Elbow Surg 2003; 12: 207-213.

14. Orfaly RM, Rockwood CA Jr and Wirth MA. A prospective functional outcome study of shoulder arthroplasty for osteo-arthritis with an intact rotator cuff. $J$ Shoulder Elbow Surg 2003; 12: 214-221.

15. Godeneche A, Boileau P, Favard L, et al. Prosthetic replacement in the treatment of osteoarthritis of the shoulder: early results of 268 cases. J Shoulder Elbow Surg 2002; 11: 11-18.

16. Raiss P, Schmitt M, Bruckner T, et al. Results of cemented total shoulder replacement with a minimum follow-up of ten years. J Bone Joint Surg Am 2012; 94: e171 (1-10).

17. Frankle M, Siegal S, Pupello D, et al. The reverse shoulder prosthesis for glenohumeral arthritis associated with severe rotator cuff deficiency. A minimum two-year follow-up study of sixty patients. $J$ Bone Joint Surg Am 2005; 87: 1697-1705.

18. Nolan BM, Ankerson E and Wiater JM. Reverse total shoulder arthroplasty improves function in cuff tear arthropathy. Clin Orthop Relat Res 2011; 464: 2476-2482.

19. Zumstein MA, Pinedo M, Old J, et al. Problems, complications, reoperations, and revisions in reverse total shoulder arthroplasty: a systematic review. J Shoulder Elbow Surg 2011; 20: 146-157.

20. Scarlat MM. Complications with reverse total shoulder arthroplasty and recent evolutions. Int Orthop 2013; 37 : 843-851.

21. Hamilton MA, Diep P, Roche C, et al. Effect of reverse shoulder design philosophy on muscle moment arms. J Orthop Res 2015; 33: 605-613.

22. Roche CP, Marczuk Y, Wright TW, et al. Scapular notching and osteophyte formation after reverse shoulder replacement: radiological analysis of implant position in male and female patients. Bone Joint $J$ 2013; 95-B: 530-535.

23. Levigne C, Boileau P, Favard L, et al. Scapular notching in reverse shoulder arthroplasty. J Shoulder Elbow Surg 2008; 17: 925-935.

24. Bacle G, Nové-Josserand L, Garaud P, et al. Long-term outcomes of reverse total shoulder arthroplasty: a followup of a previous study. J Bone Joint Surg Am 2017; 99: 454-461. 